\title{
Supporting Collaborative Learning during Information Searching
}

\author{
Michael B. Twidale, David M. Nichols, Gareth Smith and Jonathan Trevor ${ }^{*}$
}

\begin{abstract}
We consider the role of collaborative learning during information searching. We report on observations of situated collaboration in a physical library, which informed the development of our system, Ariadne. This was intended both to investigate and support the learning of search skills. An iterative development and testing methodology was applied. The system has a mechanism for recording an interaction history of the search process. A visualisation of this process makes it easier for users to reflect, share and comment upon their understanding with others.
\end{abstract}

Keywords - Information searching, collaborative browsing, iterative development, search visualisation.

\section{Introduction}

The use of library resources is stereotyped as a solitary activity with, until recently, little mention in the library science and information retrieval literature of the social aspects of information systems. However, end-users do engage in significant collaboration; both with co-searchers, library staff, professional colleagues and other interested parties $[8,13]$. The trend to increased remote access of information stores is changing the nature of social interactions in libraries and this will inevitably affect the collaboration that occurs [21].

Our approach is pragmatic rather than theory-driven as the role of collaborative learning in information searching is relatively unexplored. Similarly, the system should be regarded as much as a tool for investigating the phenomenon as it is a tool for actually supporting users. We report on observations of situated collaboration in physical libraries and the subsequent iterative development and testing of the system, Ariadne, intended to help investigate collaborative learning of search skills. We describe mechanisms both to preserve existing collaboration and to enable new forms in digital libraries.

\section{Collaboration in Information Searching}

We first consider the problems that end-users have in using information systems, and then look at the role of collaborative learning in addressing these issues.

\subsection{Information Searching}

Increasing numbers of students are using online resources including OPACs (Online Public-Access Catalogues) and bibliographic databases as part of their studies. Information searching is also becoming one of the transferable skills expected of all graduates. However, the numerous problems end-users have in effectively using online databases are well documented (e.g. [3, 29]). Common problems include: retrieving zero hits [7], retrieving hundreds of hits [11], frequent errors [2], little strategy variation [10] and locating few of the relevant records [9].

Such reports have led to a paradigm shift in the field of information science from a system-centred view of information systems to a user-centred (or 'sense-making') approach [6, 14]. This constructivist view to searching recognises that users seek information from a variety of sources to satisfy their information needs. These needs are often initially vague and evolve during the search process so that browsing is a more accurate description of users' behaviour than searching [1]. The term browsing emphasises the indeterminate, situated and serendipitous aspects of searching $[4,15,17]$ which contrast sharply with the single-query/single-answer model of traditional information retrieval [18].

Browsing is not limited to physical books or their electronic representations. That people are a valuable source of information is acknowledged in the importance placed on the user-intermediary reference interview (e.g. [5, 12, 23]). The centrality of this interaction for librarians appears to have led to an under-appreciation of the collaboration between end-users and how this can result in the collaborative learning of information skills. 


\subsection{Collaborative Learning}

Information searching is an interesting context to investigate collaborative learning because it involves two processes: learning about the domain in question (say, Psychology) and learning about how to locate information. The two processes are inextricably linked - information skills can't be effectively taught in an abstract manner. In other words, (as librarians themselves have noted) it is difficult to learn about searching for information without actually searching for some real information.

There is a notable trend, certainly in the UK University sector, for increased use of collaborative learning for two disparate reasons. The first is the belief in its educational advantages [22] including greater student enjoyment and motivation as well as greater relevance to real-world modes of working. The second is the perceived cost savings compared to conventional individual-oriented education [28].

What is remarkable about the learning of information skills is that some students spontaneously choose to collaborate. That is, unlike other forms of collaborative learning where we as educators have to set up structures to ensure collaboration occurs, in the searching context it happens independently through student-initiated actions.

Part of this spontaneous collaboration is a by-product of organised collaboration. For example, students working on a group Psychology assignment are likely to work together in the information searching stage of the activity mutually learning information searching skills. However, other instances of collaborative learning that we have observed occur independently of formally organised groups.

Inevitably, there are problems. Peers may not be able to answer all questions, some help may be confusing or simply wrong, and groups may reach impasses where they need help from outside their peer group. Therefore, any attempt to encourage collaborative learning also needs to support the occasions when it breaks down. While database browsing, the group may consult different kinds of people: other more expert peers, library staff and their subject tutor(s). All these outsiders will find the giving of help far easier if they have a context; a record of what the group has currently achieved and where problems arose [5]. As well as indicating the true source of the problem, something that novices may find particularly difficult to articulate (it is of course hard to discuss in the abstract something you know you don't understand), an activity trace also gives an expert clues about the degree of sophistication of the questioners, allowing her to phrase her explanation at the appropriate level of detail.

\section{Observations of Information Searching}

Given that it may seem counter-intuitive that library usage does indeed contain significant aspects of collaboration, we would encourage readers of this paper to spend a short time observing users in their local physical library.

Our own informal observations have shown notable work-related collaborations (excluding social chat) of roughly two every ten minutes around a bay of 12 OPAC terminals [25] and similar amounts round a group of PCs providing access to bibliographic CD-ROMs. This figure gives an indication of an order of magnitude of significance; other studies, both at Lancaster and elsewhere, are clearly required.

Several kinds of collaborative interactions between users have been observed:

- A group of students (2-4) work around a single terminal, discussing their ideas and planning their next actions. The interaction involves frequent pointing at the terminal screen. They are involved in a group task, either one set as such by their lecturer or one where they have chosen to collaborate on searching the literature before working on their individual assignments.

- A group working on adjacent terminals, discussing what they are doing, comparing results, sometimes seemingly competing to find the information. Much leaning over terminals occurs and they may occasionally all cluster around one terminal.

- Individuals working at adjacent terminals occasionally leaning over and asking their neighbour for help. These questions could be asked of a member of the library staff, but it is much more convenient (and perhaps less embarrassing) to ask a neighbour rather than to stand in a queue at the enquiries desk. In addition, the helper can see the context of the questioner's problem, something that is lost (with existing technology) if the questioner leaves her terminal.

- Individuals working at separate terminals monitoring the activity of others. There is a substantial degree of awareness while working in the library. Much of this is social (e.g. noticing friends walking past), but some appears to be an informal monitoring of the activity of others. Occasionally, this leads to a query of the form "How did you do that?". These interactions were rarer than the other kinds and occurred, unsurprisingly, most often between colleagues. 
- Patterns of work intersect at a communal resource such as a printer or a photocopier. For example, a student printing search results found an uncollected printout and inquired whose it was - when the owner was identified he proceeded to use the results to discuss the CD-ROM system.

This degree of collaboration is notable not merely because it appears to be ignored in the library and information science literature, but also because the context it occurs in might be expected to reduce the likelihood for collaboration. Libraries are perceived as quiet places where talking is frowned upon and where people go to study alone. Furthermore, bibliographic systems are designed only to support users working alone. Despite these social and technological constraints, collaborations still do occur $[8,13]$.

Nevertheless there are features that encourage collaboration. Library staff do endeavour to make libraries more welcoming [16]. In the case of Lancaster University Library, the layout promotes informal social interaction by placing communal services (help desks, photocopying, etc.) around a large public space. The OPAC terminals we observed were in this busy area.

This physicality is worthy of note because as libraries become increasingly networked and virtual, the necessity of physically working in the library will diminish [21]. A side effect will be that the existing opportunities for spontaneous co-located synchronous collaboration will likewise diminish $[13,16]$.

The move to digital libraries offers great potential for increased collaborative learning - collaborations with users remotely located but electronically linked using the same resources. However we need to ensure that our systems not only provide mechanisms to allow new forms of collaboration, but also cater for (or compensate for the loss of) the existing forms. There is a danger that this will not happen for two reasons:

- As the collaborative nature of library usage is hardly recognised in the research literature, OPAC developers are unlikely to take it into account.

- Historically, the developers of databases have attempted to make the impact of other users as near invisible as possible, thus eliminating the possibilities of collaboration [19].

\section{The Ariadne System}

Based on our studies of existing, spontaneous collaboration we have developed, and are refining, a system (Ariadne) to support the collaborative learning of database browsing skills [24].

\subsection{Aims for the System}

Ariadne is intended to serve two purposes:

- To allow us to observe, record, analyse and experiment with the collaborative learning process. Studies of the use of Ariadne such as those outlined below will provide us with more detailed information about the nature of the process we wish to support. This information will be complementary to that obtained from situated observations of collaboration in libraries.

- To provide a system which enhances the opportunities and effectiveness of the collaborative learning that already occurs. We also want to include facilities that will allow collaborations to persist as people increasingly search information remotely.

As a result of these twin aims, the development of Ariadne employs an evolutionary approach, with continual testing of the system by users. As noted, collaboration often occurs despite the features of existing systems rather than because of them. In particular, although the more sophisticated bibliographic database systems provide various options for recording (and possibly sharing) the product of a search activity (the hits obtained), none that we are aware of provide any mechanism for sharing the process (how the hits were obtained). The latter is just as important in the former in facilitating collaborative learning as the following scenarios illustrate:

- A user wishes to explain how to employ a browsing strategy to a colleague. If they happen to be in the same place a demonstration may suffice. However, having a record of the process is clearly useful, as a focus for clarifying discussion of the process and allowing the learner to take the record away with her. In asynchronous collaborations a record of the process is even more important.

- A user wishing to ask for help, can approach a colleague with a record of her process and say "I did this, what am I doing wrong?" 
- A user wishes to share with a colleague not just what they have found but how she found it. The colleague can then continue by considering which strategies have not yet been employed.

- Examples of best practice can be circulated in the learning community. Even though the topics of the actual searches may not be of relevance to the co-learners, the strategies that they embody can be, particularly if they have annotations. Just as useful can be the circulation of poor searches, coupled with annotations of what is wrong with them.

Our observations of database usage by novices and the work of others $[1,20]$ leads us to believe that one of the causes of the poor performance of novices is that they lack an overview of the browsing process. As a result they lack any systematic strategy for investigating the available information and for using interim results for refining the search process. Therefore, we believe it is important to provide a visualisation that can serve as an overview of a complete interaction history.

\subsection{Data Capture}

The system captures the users' input (key strokes) and the database/library system's output (text-based screen dumps). These are then combined to form a series of command-output pairs, each pair being represented as an item in the Ariadne browser. This means data capture is done transparently: so that users can work as if they were interacting directly with their chosen database. It is only in the subsequent playback phase that the new form of working becomes available.

The simplicity of the approach ensures that it can be used for any text based interface for any library for which remote access via Telnet is possible. This separation between capture and display is necessary because of the lack of separation between the user interface of a database and the database functionality itself. Therefore, the separation we provide allows the graphical Ariadne interface to work both with data captured in this way and (potentially) through other methods (e.g. by the Z39.50 protocol). 


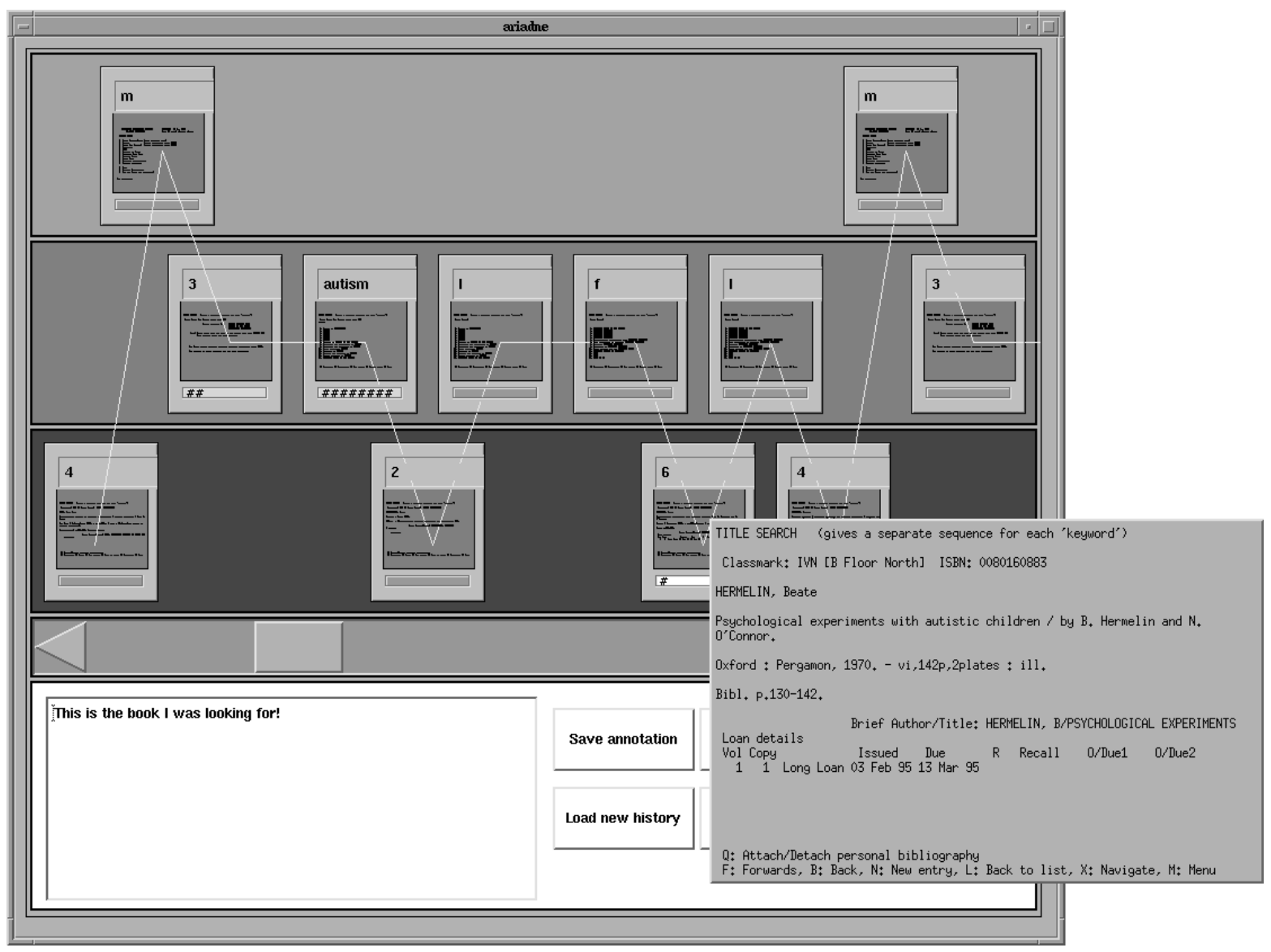

Figure 1 The Ariadne system interface (the open card has the database menu command '6' )

\subsection{Playback}

The system interprets the command-output pairs and creates a card representation for each one. Each card consists of the users' command, a thumbnail (miniaturised version) of the screendump and an annotation indicator (Figure 1). The thumbnail can be expanded by clicking on it to open a window showing the full sized view of the screenshot. The aim of the thumbnail is to act as a reminder of the underlying text. For example, it is easy to distinguish between the thumbnails of a menu screen and a database record. The interface consists of two areas, one depicting the users' browse path and one that supports annotation of the cards, separated by the scrollbar.

The browse area is sub-divided into three levels to provide a two-dimensional representation of the information searching process. The aim is to give an impression of 'diving' into a database by composing queries and going down to actual data entries. A session consists of numerous 'dives' into detail, interspersed by 'higher level' activities of composing and combining searches, selecting display options etc. For example, screen shots of top level menus are located in the top level, whereas individual book references are positioned in the lowest level. The browser employs a set of rules that define the positional semantics of the cards. Each set of rules is fixed and specific to the type of database/library system used in the search. The rules provide a first approximation to the impression of 'data diving'. Users can override the rules by moving any card up or down through the levels. The cards are placed in a 'trail'. A 'chalkline' is drawn over the trail to emphasise the chronological ordering of the search (Figure 1).

Selecting the annotation indicator box at the bottom of a card loads the annotation associated with that card (if any) into the annotation editor. Cards with annotations attached are distinguished by having a differently coloured annotation box which also displays an indication of the size of the annotation.

A user may collapse, or fold, parts of the search allowing information to be temporarily hidden. A new card is created denoting the presence of a folded region (Figure 2). Folds may be nested, annotated and expanded. The large number of cards that result from a lengthy search make it desirable to group together and hide a sequence of cards in order to focus on the larger picture. For example, a substantial part of a search may have involved looking in turn at 


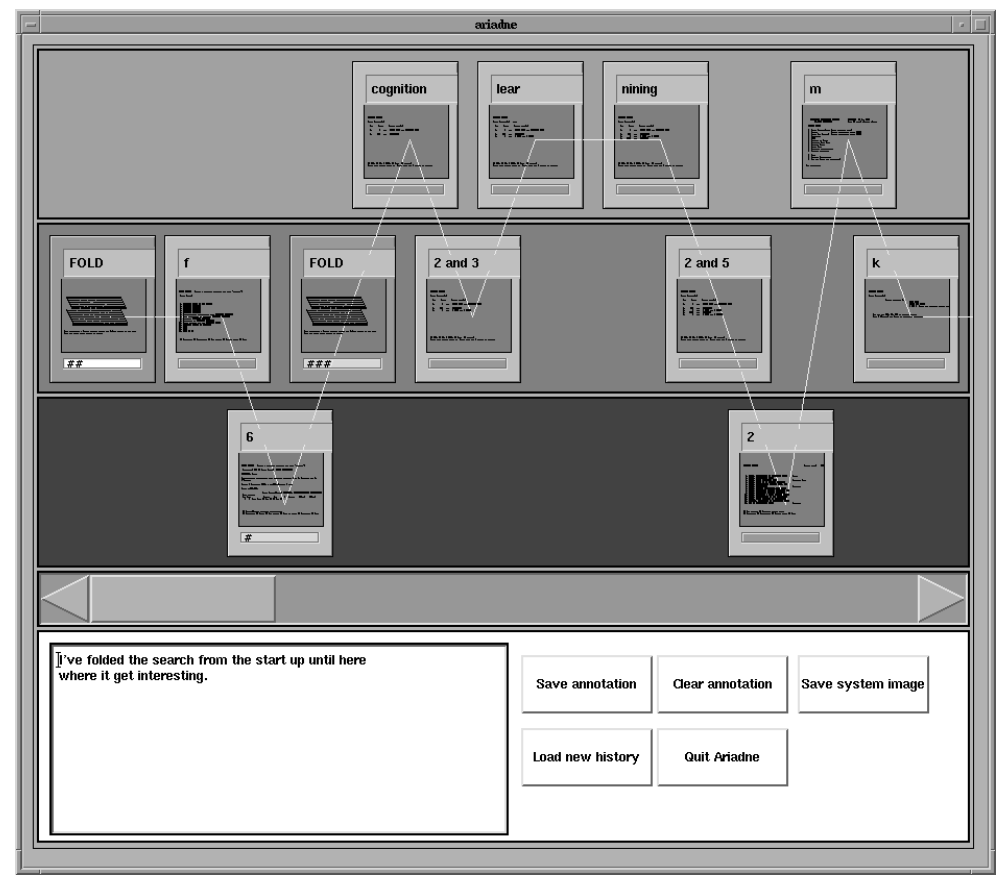

Figure 2 A search visualisation in Ariadne showing folding and annotation

a collection of, say, 23 hits. These can be folded away in order to get a better view of the other actions that took place during the search. The folds and annotations can also be saved.

Currently Ariadne can be used to record and play back interactions with the OPAC system used by Lancaster's Library and BIDS, a national online bibliographic database. In addition to revising and expanding Ariadne's functionality, we intend extending the coverage of the systems that it can support.

\section{Testing of Ariadne}

We believe that formative evaluation of the system should be as authentic as possible [26]. Therefore, testing has involved the use of volunteers who bring a problem that they already have to solve, rather than our imposing a standardised problem upon them. The latter approach has many problems, not least that the implicit assumptions in the design of the system are likely to also be manifested in the design of the activity to be performed in the evaluation, thus reducing the chances of detecting those assumptions, and of revealing the requirements of actual users.

Given the incremental development of Ariadne, the results of the sequence of testing are necessarily both preliminary and anecdotal. Nevertheless we believe that the results obtained are of importance in informing subsequent development of the system and in contributing to our understanding of the nature of the behaviour we wish to support [27].

\subsection{Collaborative Use of Ariadne}

Five groups of four undergraduate Psychology students chose to use the system to help them with their group assignment. They had to agree a general theme in Cognitive Psychology and then each student had to pick a more precise topic to study in detail from the primary literature. They were each to produce an individual report which would be collected together and, with an initial linking chapter, would form their coursework document. The students used Ariadne (largely with BIDS) at the beginning of the task, both to decide on their collective theme and on their individual subtopics. This activity revealed one of the key characteristics of browsing; how the goals evolve over time. The process of analysing retrieved hits from a search led the groups to redefine what it was that they wished to investigate. Some groups chose to return to continue working in later sessions, as individuals, subgroups and full groups. Most students were unfamiliar with bibliographic databases but confident in using the basic options of Lancaster's OPAC.

An initial concern was that the concept of visualising a search process might require a certain degree of understanding of the nature of bibliographic database use. For competent users it is fairly clear that a record of their 
actions would allow them to reflect on what they have done, share and discuss it with colleagues and consider alternatives and improvements. What was less clear was whether the system would be of use to relative novices.

\subsection{Comprehension of the Search Visualisation}

Users were able to understand the 2D visualisation of the search. They were happy to scroll over their earlier searches, opening up screens to examine in detail the what they had done and use that in group discussions of what they should do next, and how they should do it.

This is encouraging, given that :

- None of them had ever experienced any visualisation of search process before.

- Some were very unfamiliar with computer use, particularly graphical workstations.

- Many had only a very rudimentary understanding of the nature of information browsing in databases, and the strategies and tactics that can be applied.

\subsection{Observations on Ariadne Usage}

The history representation became a focus for discussion. This has been noted for other CSCW and CSCL systems [27]. For those groups who chose to return for subsequent sessions, the ability to rapidly review the search they performed a week or more ago proved to be a useful orientation activity, particularly where the group members had not convened in other circumstances since the last session.

Users were keen to get printouts of information they had found. Consequently an option to print the contents of a card was added. Numerous other minor changes to the interface were made in the light of the iterative testing and development. Although seemingly minor, interface issues such as the positioning and wording of menu options can have a enormous effect on usability for novices. A consequence of this is that the folding and annotation facilities have received less testing as they have evolved rapidly. Most of their use has so far been by the experimenter when acting as a domain expert, as noted below.

Groups of novice users inevitably hit problems which they could not solve collaboratively. In such cases, when asked, the experimenter intervened and acted as an information intermediary (such as a subject librarian). Ariadne's visualisation proved particularly useful for explaining and summarising to the group what they had done and to initiate discussion of what they might try next.

Many mistakes were made in searching. These can be caused by substantial misconceptions about the nature of bibliographic databases [29], but also by simple typing errors. The latter are of concern because of occasions when a group attempted a perfectly sound strategy, but due to a minor error (a typing or syntactic mistake), the action failed to yield anything and so the strategy was abandoned. The users may not be aware of the error and, we observed in some cases, refused to believe that they had made it until confronted with the evidence of the history on the screen.

Searches can be performed but not investigated. That is, the users undertake a search and get a reasonable number of hits, but fail to look at these hits, not even listing their titles. Instead they go on and do another search, and in the cases we note here, never go back to examine the results. This can be due to the presence of multiple search goals, so that completing one leads to attempting the next before the results of the first are examined. Another observed cause was that undertaking one search inspired an idea for another tactic, and the users got carried away with trying out this technique before examining the results of their earlier trial. This behaviour has been noted both for individuals and for groups. We believe the situation may be more acute for groups, where a number of participants have competing suggestions for the next search tactic to try. The ability to subsequently view their actions using Ariadne proved useful to some groups in revealing this problem, and in inspiring group members to suggest subsequent search activities. Other groups were not aware of gaps in their searching until the experimenter intervened.

\section{Future Work}

So far, all the testing of Ariadne has been synchronous and co-located. We have also undertaken small scale studies of other modes of cooperative working [24] which indicated problems with the usefulness of synchronous remote collaboration. Therefore, we are developing Ariadne to support asynchronous, remote collaboration. One aspect of this will be the sending of annotated searches using electronic mail. In order to investigate another approach, we have already implemented an option to convert the record of a search into a sequence of World Wide Web (WWW) pages. Examples can be found at: http://www.comp.lancs.ac.uk/computing/research/aai-aied/information/ariadne/ 


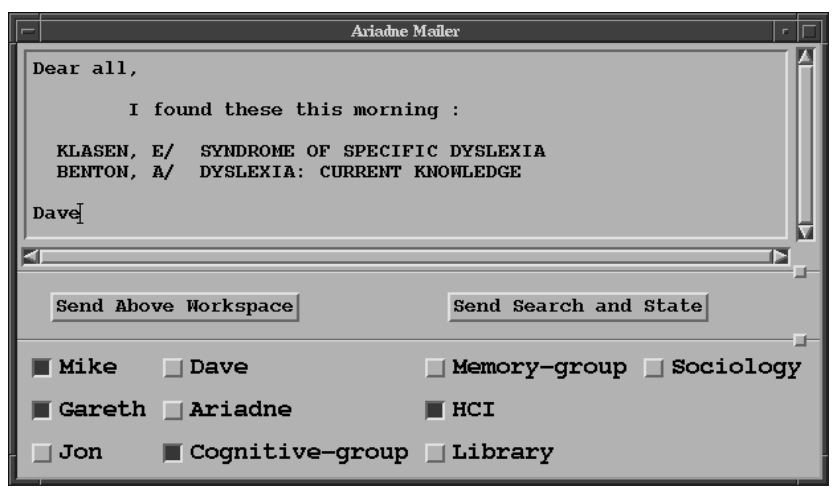

Figure 3 The Ariadne collaborative window

\subsection{New Functionality}

The latest version of Ariadne also has a collaborative window to support asynchronous communication (Figure 3). The thumbnails of the cards on the main browsing window may be selected and dragged over to the text editor of the collaborative window and dropped into it. The toggle buttons (whose details are obtained from the user's own resource file) denote users and groups who are to receive the message. A user may communicate with a number of other users and groups by selecting an appropriate combination of toggle buttons. Two modes of communication are possible:

- The contents of the text editor may be sent. This utilises a normal email service which allows users to extract the text based information held within the Ariadne browser.

- The user can send her current search in its existing state. This can then be visualised, further annotated and returned or forwarded to others.

We believe that if we are to facilitate the widest possible degree of collaboration we need to allow for a range of technologies and platforms. Although closely cooperating group members may all be using the same technology, they may wish to draw on the assistance of an outsider who does not have the same systems available. Hence the group may use Ariadne to cooperate between themselves, but have to resort to the WWW histories or conventional email for working with others. Collaborative systems should be designed to support a graceful degradation of functionality to permit a wider degree of participation.

\section{Conclusion}

We have described how the computer supported collaborative aspects of learning information searching skills have been relatively ignored. Students already use libraries as locations to undertake collaborative learning. We believe it to be important to provide users with mechanisms that allow them to share the process of their searching activities as well as its product, and also for the systems to facilitate an awareness of others. Our system, Ariadne, allows us to investigate how to support existing collaboration and to address the challenges and opportunities that the transition to digital libraries affords.

\section{Acknowledgements}

Financial support for this research was provided by the HEFCs' JISC New Technologies Initiative.

\section{References}

1. Bates, M.J. (1989), The design of browsing and berrypicking techniques for the online search interface, Online Review, 13(5), 407-24. 
2. Borgman, C.L. (1983), End user behavior on an online information retrieval system: a computer monitoring study, ACM SIGIR Forum, 17(4), 162-76.

3. Borgman, C.L. (1986), Why are online catalogs hard to use? Lessons learned from information retrieval studies, Journal of the American Society for Information Science, 37(6), 387-400.

4. Chang, S.J. \& Rice, R.E. (1993), Browsing - a multidimensional framework, Annual Review of Information Science and Technology, 28, 231-76.

5. Dervin, B. \& Dewdney, P. (1986), Neutral questioning: a new approach to the reference interview, Reference Quarterly, 25(4), 506-13.

6. Dervin, B. \& Nilan, M.S. (1986), Information needs and uses, Annual Review of Information Science and Technology, 21, 3-33.

7. Dickson, J. (1984), An analysis of user errors in searching an online catalog, Cataloging and Classification Quarterly, 4(3), 19-38.

8. Erlich, K. \& Cash, D. (1994), Turning information into knowledge: information finding as a collaborative activity, Proceedings of Digital Libraries '94, College Station, TX, 119-25.

9. Fenichel, C.H. (1980), The process of searching online bibliographic databases: a review of research, Library Research, 2(2), 107-27.

10. Fenichel, C.H. (1981), Online searching: measures that discriminate among users with different types of experience, Journal of the American Society for Information Science, 32(1), 23-32.

11. Graham, T. (1985), The free language approach to online catalogues: the user, in Bryant, P. (Ed.), Keyword Catalogues and the Free Language Approach, Bath, UK: Bath University Library,

12. Kuhlthau, C., Spink, A. \& Cool, C. (1992), Exploration into stages in the information search process in online information retrieval, Proceedings of ASIS Annual Meeting (Vol. 29), 67-71.

13. Levy, D.M. \& Marshall, C.C. (1995), Going digital: a look at assumptions underlying digital libraries, Communications of the ACM, 38(4), 77-84.

14. Morris, R.C.T. (1994), Toward a user-centered information service, Journal of the American Society for Information Science, 45(1), 20-30.

15. O'Connor, B. (1993), Browsing: a framework for seeking functional information, Knowledge: creation, diffusion, utilization, 15(2), 211-32.

16. Reich, V. \& Weiser, M. (1993), Libraries are more than information: situational aspects of electronic libraries, Serials Review, 20(3), 31-7.

17. Rice, J. (1988), Serendipity and holism: the beauty of opacs, Library Journal, 113(3), 138-41.

18. Robertson, S.E. (1977), Theories and models in information retrieval, Journal of Documentation, 33(2), 12648.

19. Rodden, T., Mariani, J.A. \& Blair, G.S. (1992), Supporting cooperative applications, Computer Supported Cooperative Work, 1(1), 41-67.

20. Rudd, J. \& Rudd, M.J. (1986), Coping with information load: strategies and implications for librarians, College \& Research Libraries, 47(4), 315-22.

21. Shaw, D. (1994), Libraries of the future: glimpses of a networked, distributed, collaborative, hyper, virtual world, Libri, 44(3), 206-23. 
22. Sheridan, J. (1990), The reflective librarian: some observations on bibliographic instruction in the academic library, Journal of Academic Librarianship, 16(1), 22-6.

23. Taylor, R.S. (1968), Question-negotiation and information seeking in libraries, College \& Research Libraries, 29(3), 178-94.

24. Twidale, M.B., Mariani, J.A., Rodden, T., Sawyer, P. \& Nichols, D.M. (1995), An interface to support collaborative database browsing, Paper presented at CAL '95, Cambridge, UK.

25. Twidale, M.B. \& Nichols, D.M. (1995), Situated Observations of Library Users, Internal Report, Computing Department, Lancaster University.

26. Twidale, M.B., Randall, D. \& Bentley, R. (1994), Situated evaluation for cooperative systems, Proceedings of CSCW'94, Chapel Hill, NC, ACM Press, 441-52.

27. Twidale, M.B., Rodden, T. \& Sommerville, I. (1994), The use of a computational tool to support the refinement of ideas, Computers and Education, 22(1/2), 107-18.

28. Warmkessel, M.M. \& Carothers, F.M. (1993), Collaborative learning and bibliographic instruction, Journal of Academic Librarianship, 19(1), 4-7.

29. Yee, M.M. (1991), System design and cataloguing meet the user, Journal of the American Society for Information Science, 42(2), 78-98.

\section{Authors' Addresses}

Michael B. Twidale, David M. Nichols, and Gareth Smith: Computing Department, Lancaster University, Lancaster LA1 4YR, UK. \{mbt, dmn, gbs\}@ comp.lancs.ac.uk;

Jonathan Trevor: GMD-FIT.CSCW, Schloß Birlinghoven, D-53754 Sankt Augustin, Germany. trevor@gmd.de.

Twidale, M.B., Nichols, D.M., Smith, G. and Trevor, J. (1995) Supporting collaborative learning during information searching. Proceedings of the First International Conference on Computer Support for Collaborative Learning (CSCL '95), L. Erlbaum Associates Inc., Hillsdale, NJ, USA. 367-374. 